\title{
LETTER TO THE EDITOR Cryo-EM structure of the human mitochondrial translocase TIM22 complex
}

\author{
Cell Research (2021) 31:369-372; https://doi.org/10.1038/s41422-020-00400-w
}

Dear Editor,

Mitochondria are essential organelles in cellular metabolism, homeostasis, and apoptosis. ${ }^{1,2}$ Most mitochondrial proteins are synthesized as precursors in the cytosol and then imported into mitochondria by specific protein translocase complexes, including the translocase of the outer membrane complex (TOM complex), the carrier translocase of the inner membrane complex (TIM22 complex), the presequence translocase of the inner membrane complex (TIM23 complex), the sorting and assembly machinery (SAM complex), and the mitochondrial import complex (MIM complex). ${ }^{3}$ The TIM22 complex is responsible for the translocation and insertion of hydrophobic membrane proteins, including mitochondrial carrier proteins and translocase subunits (Tim17, Tim22 and Tim23). ${ }^{3}$ In humans, TIM22 is a $440-\mathrm{kDa}$ complex comprising at least six components: the hypothetical channelforming protein Tim22, three small Tim proteins (Tim9, Tim10a and Tim10b), Tim29 and acylglycerol kinase (AGK). ${ }^{1}$ Considering the functional importance of mitochondrial protein import, the TIM22 complex has been linked to many diseases. For example, mutations in the TIM22 gene have been reported to cause earlyonset mitochondrial myopathy. ${ }^{4}$ AGK participates in lipid biosynthesis, and mutations in the $A G K$ gene lead to Sengers syndrome. ${ }^{2}$ Mutations in the TIMM $8 A$ gene (also called DDP1) cause deafness dystonia syndrome. ${ }^{2}$

Despite advances in our knowledge of the function and pathophysiology of the TIM22 complex, reports of its structural characterization are scarce. The structural studies of the TIM22 complex are restricted to the investigation of the structures of Tim9/10 ${ }^{5,6}$ and Tim9/10/12 hexameric chaperone ${ }^{7}$ and a nuclear magnetic resonance (NMR) analysis of carrier precursors associated with the Tim9/Tim 10 complex. ${ }^{8}$ Here, we report the cryoEM structure of the human TIM22 complex at an overall resolution of $3.7 \AA$.

We coexpressed all six known components of the TIM22 complex in human embryonic kidney (HEK) $293 \mathrm{~F}$ cells (Fig. 1a). After Flag tag affinity purification followed by gel filtration, the resultant TIM22 complex displayed good resolution behavior (Fig. 1b). The apparent molecular weight was assessed by blue native PAGE to be approximate $440 \mathrm{kDa}$ (Supplementary information, Fig. S1a), consistent with previous findings. ${ }^{9-12}$ Mass spectrometry (MS) analysis of the purified complex confirmed the presence of all known components of the TIM22 complex. Furthermore, mitochondria from cells overexpressing the TIM22 complex exhibited more efficient carrier-importing activity than those from wild-type cells (Supplementary information, Fig. S1b, c).

The details of grid preparation, cryo-EM data acquisition, and structural determination of the TIM22 complex are presented in "Supplementary information, Materials and Methods". The initial 2D classification, 3D classification and refinement of the cryo-EM particle images yielded a final 3D EM reconstruction map at an overall resolution of $3.7 \AA$ and an intermembrane region resolution of $3.5 \AA$ (Fig. 1c; Supplementary information, Fig. S2 and Table S1). Atomic models were built into the map for Tim22, Tim9, Tim10a, Tim10b, Tim29 and AGK (Supplementary information, Fig. S3 and Table S2; examples of local densities are shown in Supplementary information, Fig. S4). Since the density of the Tim29 N-terminal helix was poor, poly-Ala was assigned to this region.

The overall structure of the TIM22 complex was approximately $100 \AA$ in height and $160 \AA$ in width (Fig. 1d). The structure contained one Tim22 molecule, one Tim29 molecule, one AGK molecule, and two hexamer chaperones, Tim9/10a (3:3) and Tim9/ $10 \mathrm{a} / 10 \mathrm{~b}$ (2:3:1) (Fig. 1d). Most regions of the structure, including the N-terminus of Tim22 and the extended C-terminal portion of Tim29, were located in the intermembrane space (IMS) (Supplementary information, Fig. S5a). Four transmembrane segments (TMs) of Tim22 and a single TM of Tim29 and AGK constitute the transmembrane region at the center of the TIM22 complex (Fig. 1d). The N-terminal helix of Tim29 protrudes from the core transmembrane region, and it is near and horizontal to the membrane plane on the matrix side. Similar to a hub, the Tim9/ $10 \mathrm{a} / 10 \mathrm{~b}$ hexamer was encircled by the N-terminus of Tim22, the middle portion of Tim29, AGK, and the Tim9/10a chaperone (Fig. 1d). The Tim9/10a/10b hexamer was not perpendicular to the membrane but instead was tilted approximately $45^{\circ}$. The Tim $9 / 10 \mathrm{a}$ chaperone and AGK were located on nearly opposite sides of the Tim9/10a/10b hexamer, and AGK was anchored to the inner membrane via its portion of the $\mathrm{N}$-terminal helix and an additional membrane anchor (helix-loop-helix) (Fig. 1d; Supplementary information, Fig. S5a, b).

Tim22 contains 4 TMs (TM 1-4) and two helices (a1 and a2) connected by extended loop 1 (Fig. 1e). The $\mathrm{N}$-terminus (residues 1-23) and the matrix loop (residues 94-118; connecting TM1 and TM2) exhibited no electron density, possibly because of their intrinsic flexibility. A disulfide bond formed between Cys69 and Cys141 seemed to stabilize the conformation of TM1 and TM2 (Supplementary information, Fig. S6a). This observation was consistent with previous findings that the disulfide bonds play important roles in the assembly of the TIM22 complex. ${ }^{10}$ Helix a1 and loop 1, like a hook, sinuously winded around the conserved hydrophobic groove between the inner helices and the outer helices of Tim 9 and Tim10a (Supplementary information, Fig. S6b). Helix a 1 was nestled in a greasy pocket formed by Tim9 and Tim10a and possibly functioned as a plug to obstruct the hydrophobic carrier precursor from wedging into the hexamer chaperone through this side (Fig. 1e; Supplementary information, Fig. S6b). Moreover, the recently identified disease-related mutation (Val33Leu) of Tim22 was located in helix a1. ${ }^{4}$

These interactions comprised van der Waals contacts and hydrogen bonds. The hydrophobic residues Leu28, Leu29, Leu32, and Val33 in the N-terminal plug of Tim22 interacted with the Leu9, Leu13, Met17, and Leu69 residues in the helices of Tim10a and the Phe14, Phe17, and Leu18 residues in the inner helix of 
a

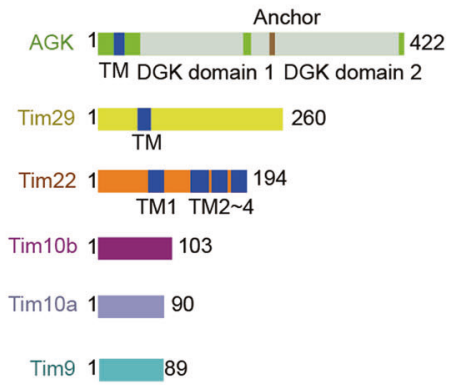

b

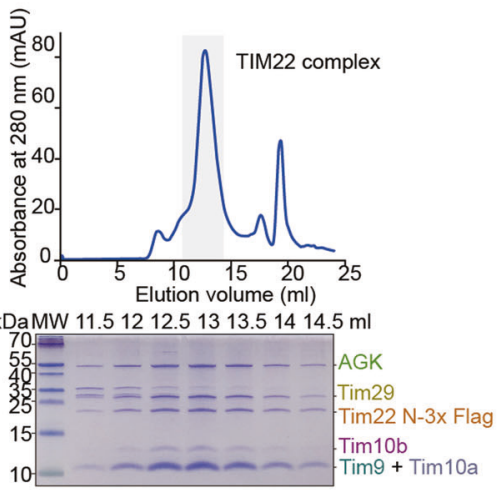

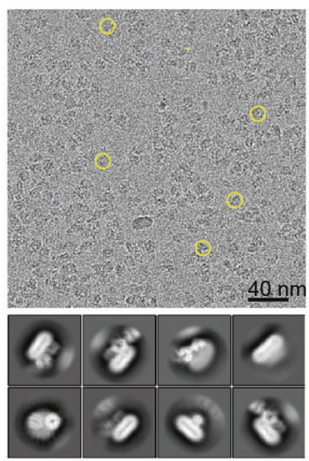

d
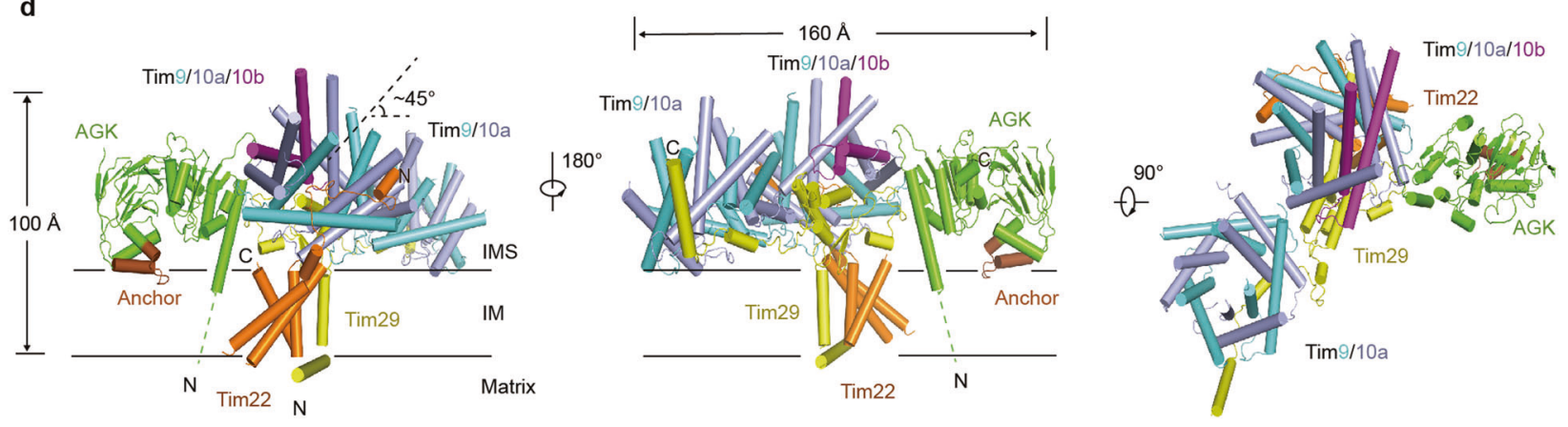

e
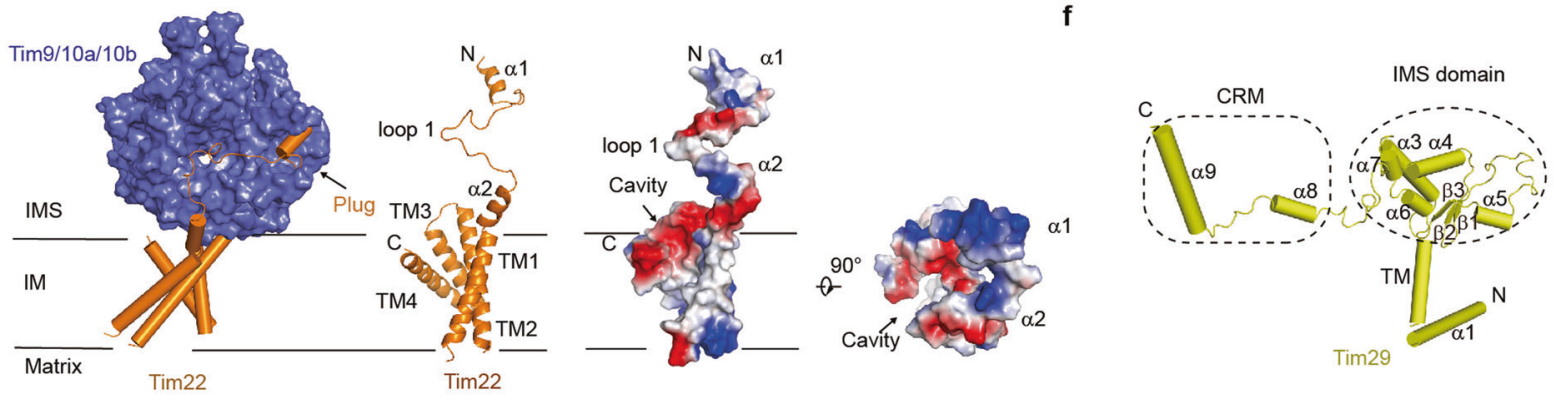

g

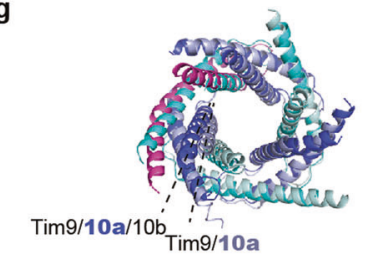

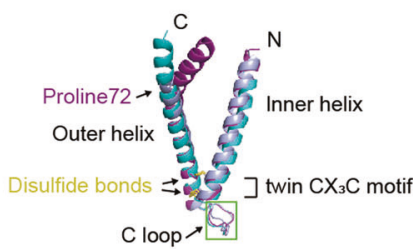

c loop

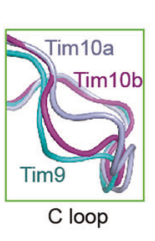

Outer helix

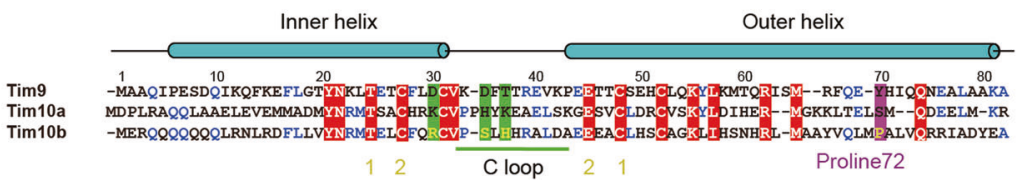

h

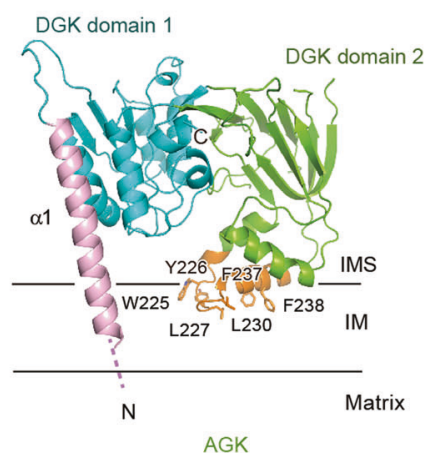

Tim9 (Supplementary information, Fig. S6b). The aromatic ring of Tyr25 in Tim22 stacked against those of Phe14 and Phe17 in Tim9. The side chain of Asn75 in Tim9 formed a hydrogen bond with the backbone of Arg40 in Tim22 (Supplementary information, Fig. S6b). Four residues from the last turn of TM2 in Tim22 contacted the residues in the outer helix of Tim10a to reinforce the abovementioned interactions. The side chains of Glu144 and Ser150 in Tim22 formed hydrogen bonds with the side chain of
Ser44 and the backbone of Gly46 in Tim10a. In addition, the backbone of Ser145 and Tyr146 in Tim22 formed hydrogen bonds with the side chain of Arg53 in Tim10a (Supplementary information, Fig. S6b). The integrity of the TIM22 complex was completely abolished by mutation of the hydrophobic residues in the Tim22 plug to Asn residues and by mutation of the hydrophilic residues in TM2, suggesting the critical role of Tim22 in complex assembly (Supplementary information, Figs. S7, S8a). Furthermore, 
Fig. 1 Cryo-EM structure of the human TIM22 complex. a The schematic diagram for each subunit of the TIM22 complex. b A representative gel filtration chromatography of the TIM22 complex. The peak fractions are pooled for cryo-EM study. $\mathbf{c}$ A representative cryo-EM micrograph and 2D class averages images of the TIM22 complex. The typical particles are marked by yellow circles. Scale bar, $40 \mathrm{~nm}$. $\mathbf{d}$ The opposing side views and top view of the TIM22 complex. The inner membrane (IM) is indicated by two lines between the intermembrane space (IMS) and the matrix. The $\mathrm{N}$ - and C-terminus of each subunits of the TIM22 complex are labeled. The membranes anchor motif of AGK is highlighted in brown. Tim9, cyan; Tim10a, slate; Tim10b, magenta; Tim22, orange; Tim29, yellow; AGK, chartreuse; All structure figures are prepared using PyMol. e Structure of the Tim22. A structural display of Tim22 in side view as cartoon. The Tim9/10a/10b hexamer is shown in surface model. The surface electrostatic potential of Tim22 is calculated using PyMOL, which shows the cavity. $\mathbf{f}$ The overall structure of the Tim29. The C-terminus of Tim29 is divided into two segments: IMS domain and CRM (C-terminal recruiting motif). The secondary structural elements are labeled. g Organization of the Tim9/10a and Tim9/10a/10b hexamer. A structure alignment of Tim9/10a/10b with Tim9/10a is shown as cartoon. Disulfide bonds formed from the signature cysteines of the twin $\mathrm{CX}_{3} \mathrm{C}$ motif are indicated. The $\mathrm{C}$ (center) loop is highlighted by a green box. Residues in the C loop involved in the interaction with the Tim 29 subunit are colored green. $\mathbf{h}$ AGK is composed of $\alpha 1$, DGK domain 1 and DGK domain 2. Key residues bound to the membrane are shown as orange sticks. DGK, diacylglycerol kinase.

mutation of the hydrophobic residues in Tim9 and Tim10a to Asn resulted in a decreased amount of the chaperone in the complex and seriously affected the assembly of the TIM22 complex (Supplementary information, Figs. S7, S8d, e). Additionally, Tim22 homologs from yeast to human shared $29 \%$ identity, with conservation of most interacting residues and two cysteines (Supplementary information, Fig. S6c), suggesting that the Tim22 homologs exhibit similar folds.

Only one Tim22 molecule was observed in the complex. The four TMs of Tim22 constituted a lateral cavity that was exposed to the lipid bilayer (Fig. 1e). Furthermore, part of the cavity near the IMS was negatively charged, and the region of this cavity proximal to the matrix was rich in hydrophobic residues (Supplementary information, Fig. S9a). Since the bacterial YidC insertase is responsible for protein translocation and membrane integration, ${ }^{13}$ we compared its transmembrane domain with that of Tim22. YidC functioned as a monomer; its five TMs created a positively charged hydrophilic cavity that opened towards the membrane and the cytoplasm, whereas the extracellular side of the cavity was sealed by the hydrophobic core (Supplementary information, Fig. S9b). ${ }^{13}$ These observations suggested that Tim22 might functioned similar to the YidC insertase family, although this hypothesis requires more structural and functional evidences.

Tim29 exhibited an extended conformation comprising a long $\mathrm{N}$-terminal helix a1 in the matrix, a single TM, an IMS domain and a C-terminal recruiting motif (CRM) (Fig. 1f). A phospholipid molecule, which was tentatively annotated phosphatidylethanolamine, was observed at the IMS domain and was speculated to stabilize the conformation of Tim29 (Supplementary information, Fig. S10a). The helix a1 was near and horizontal to the plane of the membrane in the matrix and perpendicular to the TM of Tim29 (Supplementary information, Fig. S10b), possibly interacting with TM3 of Tim22. However, the lack of electron density in the side chains of the a1 helix precluded an unambiguous annotation of the details of these molecular interactions. Deletion of a1 helix had little effect on the integrity of the TIM22 complex (Supplementary information, Figs. S7, S8b). The single TM of Tim29 was positioned far from the TMs of Tim22, suggesting the absence of a strictly association between these TMs (Supplementary information, Fig. S10b). This arrangement suggested that the TM may be flexible to promote the regulation of precursor insertion.

Tim29 acted as a scaffold to organize all other components. It held Tim22 in proximity to the Tim9/10a/10b hexamer and AGK via the IMS domain. The extended CRM interacted with the Tim9/ 10a chaperone to position Tim9/10a for transfer of the precursor to the Tim9/10a/10b hexamer (Supplementary information, Figs. S5a, S10b). The IMS domain of Tim29 interacted with the Tim9/10a/10b hexamer mainly through the a4 helix. More specifically, three polar residues Glu107, Gln111, and Arg119 in the a4 helix of Tim29 formed hydrogen bonds with His37, Ser35, and Arg31 of Tim10b, respectively (Supplementary information, Figs. S10c, S11). Additionally, a hydrogen bond was formed between the backbones of Ser143 in Tim29 and Lys32 in Tim10a. These interactions securely fixed the orientation of the Tim9/10a/ $10 \mathrm{~b}$ hexamer, partially due to the presence of only one Tim10b molecule in the heterohexamer (Supplementary information, Figs. S5a, S10b). The double mutant Glu107Lys/GIn111Ala in Tim29 moderately disrupted the interactions with the chaperone (Supplementary information, Figs. S7, S8b). The CRM of Tim29 made contact with the Tim9/10a chaperone via extensive electrostatic interactions (Supplementary information, Fig. S10b). The negatively charged residues Asp183, Asp186, Glu210, and Asp214 interacted with several positively charged residues, including Arg39 in Tim9 and Arg53, Lys 45, Lys32, and Arg31 in Tim10a (Supplementary information, Fig. S10c). In addition, neither the CRM deletion mutant ( $\triangle$ Glu188-Arg260) nor mutants of interacting residues in Tim29 and Tim10a could efficiently recruit the Tim9/10a chaperone and assemble the TIM22 complex (Supplementary information, Figs. S7, S8b, e). Taken together, our structural evidence supported previous results indicating that Tim29 is essential for the stability and assembly of the TIM22 complex. ${ }^{9,10}$

The Tim9/10a chaperone and the Tim9/10a/10b chaperone shared symmetric donut-shaped hexamer structures almost identical to those of the reported free Tim9/10a hexamer ${ }^{5}$ (Fig. 1g; Supplementary information, Fig. S12a-c). Each subunit contained a helix-loop-helix fold and was stabilized by two intramolecular disulfide bonds from a highly conserved "twin $\mathrm{CX}_{3} \mathrm{C}$ motif" (Fig. 1g). Superimposition of Tim9, Tim10a, and Tim10b revealed that the less-conserved connecting loop (C loop) exhibited a distinct conformation (Fig. 1g), consistent with our finding that the $\mathrm{C}$ loop of Tim 10b was primarily responsible for its interaction with Tim29 (Supplementary information, Fig. S10b). Furthermore, the Cterminus of the outer helix of Tim10b tilted towards the inner helix due to its Pro72 residue (Fig. 1g). The diameter of the Tim9/10a hole was slightly narrowed (Supplementary information, Fig. S12d). The major conformational difference between these two chaperones might be attributed to the outer helix of Tim10b. A circular groove that was previously hypothesized to hold unstructured carrier precursors was observed between the inner and outer helices. ${ }^{8}$ In addition, the twisted outer helix of Tim10b disrupted the continuous groove (Supplementary information, Fig. S12c). Accordingly, we speculated that this outer helix might be involved in precursors unloading from the Tim9/10a chaperone.

AGK is a mitochondrial lipid kinase that converts monoacylglycerol (MAG) and diacylglycerol (DAG) to lysophosphatidic acid (lyso-PA) and phosphatidic acid (PA), respectively. ${ }^{14}$ In this study, the AGK structure exhibited a typical two-domain fold (Fig. $1 \mathrm{~h}$; Supplementary information, Fig. S13a). Part of a predicted TM (a1 helix) preceding domain 1 was observed (Fig. 1h), consistent with previous findings. ${ }^{11,12}$ A protruding helix (a9) and an ensuing loop of domain 2 were anchored to the membrane via Trp225, Tyr226, Leu227, Leu230, Phe237, and Phe238 (Fig. 1h). Consistent with sequence alignment (Supplementary information, Fig. S14), these membrane-anchoring structural features were AGK-specific. An 
Asparagine substitution mutation in the anchor region affected the assembly of the TIM22 complex (Supplementary information, Figs. S7, S8c). Furthermore, AGK specifically interacted with both Tim29 and the Tim9/10a/10b hexamer (Supplementary information, Fig. S13b). Arg40 in the a1 helix formed hydrogen bonds with the backbones of Tyr151 and Gln153 in Tim29. Gln52, Ala58, and Asp94 were found to form hydrogen bonds with Lys45 in Tim10a, Arg62 in Tim10b, and Arg39 in Tim9, respectively (Supplementary information, Fig. S13c). These features corroborate findings from previous studies suggesting that AGK is a bona fide subunit of the TIM22 complex. ${ }^{11,12}$

Notably, the composition of human TIM22 complex differs from that of the yeast complex, which is a $300-\mathrm{kDa}$ complex consisting of four membrane integral subunits (Tim22, Tim54, Tim18, and Sdh3) and a peripheral chaperone subcomplex (Tim9/10/12). ${ }^{1,3}$ Only Tim22 and small Tim proteins were conserved from yeast to mammals, while homologs of Tim18 and Tim54 are absent in metazoans. ${ }^{1}$ Previous negative stain electron microscopy analysis suggested that the yeast TIM22 complex is a twin-pore translocase. ${ }^{15}$ This two-pore feature might be constituted by two hexamers (Tim9/Tim10 and Tim9/Tim10/Tim12), as shown in the TIM22 complex. However, a distinct architecture of the yeast TIM22 complex cannot be excluded.

In summary, we determined the cryo-EM structure of human TIM22 complex. The structure revealed the assembly and detailed structural information of the complex and provided an important framework for understanding the function and mechanism of this complex in carrier protein insertion. Previous biochemical studies have demonstrated that Tim29 also interacts physically with the TOM complex to mediate the transfer of the carrier precursor to the TIM22 complex. ${ }^{9}$ These interactions might facilitate the transfer of the carrier precursor from the Tom40 channel to the Tim9/10a chaperone in TIM22 to prevent aggregation. Afterwards, the precursor might undergo insertion into the cavity formed by Tim22 in a membrane potential-dependent manner followed by lateral release to the inner membrane (Supplementary information, Fig. S15). However, the elaborate structures of the TIM22 complex with the carrier precursor during an insertion cycle are highly important for gaining a comprehensive understanding of the carrier translocation mechanism.

\section{DATA AVAILABILITY}

The cryo-EM density map for the human TIM22 complex has been deposited in EM Database under the accession code EMD-9958. The corresponding atomic coordinates have been deposited in the Protein Data Bank under accession code 7CGP.

\section{ACKNOWLEDGEMENTS}

We thank Dr. Chang at Center of Cryo Electron Microscopy, Zhejiang University, for assistance during data collection; and research associates at the Center for Protein Research and Public Laboratory of Electron Microscopy, Huazhong Agricultural University, for technical support. We thank the Tsinghua University Branch of China National Center for Protein Sciences (Beijing) for providing the technical support on the Cryo-EM and High-Performance Computation platforms. We thank Meng Han and protein chemistry Facility at the Center for biomedical Analysis of Tsinghua University for sample mass-spec analysis. We thank Ms. Wang Yan for model figure design. This work was supported by funds from the Ministry of Science and Technology of China (2018YFA0507700), the National Natural Science Foundation of China (31722017), the Fok Ying-Tong Education Foundation (151021), and the Fundamental Research Funds for the Central Universities (2662017PY031). This research was supported by Beijing Advanced Innovation Center for Structural Biology (to Dr. Chuangye Yan).

\section{AUTHOR CONTRIBUTIONS}

P.Y. conceived the project. L.Q., Q.W., P.Y. designed all experiments. L.Q., Q.W., Y.W., C.S., and S.H performed the experiments. Q.W., Z.G., J.C., and X.Z. collected the EM data. Z.G. and C.Y. determined the structure. All authors analyzed the data and contributed to manuscript preparation. P.Y. wrote the manuscript.

\section{Competing interests}

The authors declare no competing interests.

Liangbo $\mathrm{Qi}^{1}$, Qiang Wang ${ }^{1}$, Zeyuan Guan ${ }^{1}{ }^{1}$, Yan $\mathrm{Wu}^{1}$, Cuicui Shen ${ }^{1}$, Sixing Hong ${ }^{1}$, Jianbo $\mathrm{CaO}^{2}$, Xing Zhang ${ }^{3}$, Chuangye Yan (iD ${ }^{4}$ and Ping Yin (iD)

${ }^{1}$ National Key Laboratory of Crop Genetic Improvement and National Centre of Plant Gene Research, Huazhong Agricultural University, Wuhan, Hubei 430070, China; ${ }^{2}$ Public Laboratory of Electron Microscopy, Huazhong Agricultural University, Wuhan, Hubei 430070, China; ${ }^{3}$ Department of Biophysics, and Department of Pathology of Sir Run Run Shaw Hospital, Zhejiang University School of Medicine, Center of Cryo Electron Microscopy, Zhejiang University, Hangzhou, Zhejiang 310058, China and ${ }^{4}$ Beijing Advanced Innovation Center for Structural Biology, Tsinghua-Peking Joint Center for Life Sciences,

School of Life Sciences, Tsinghua University, Beijing 100084, China These authors contributed equally: Liangbo Qi, Qiang Wang, Zeyuan Guan

Correspondence: Chuangye Yan (yancy2019@mail.tsinghua.edu.cn) or Ping Yin (yinping@mail.hzau.edu.cn)

\section{REFERENCES}

1. Kang, Y., Fielden, L. F. \& Stojanovski, D. Semin. Cell Dev. Biol. 76, 142-153 (2018).

2. Pfanner, N., Warscheid, B. \& Wiedemann, N. Nat. Rev. Mol. Cell Biol. 20, 267-284 (2019).

3. Wiedemann, N. \& Pfanner, N. Annu. Rev, Biochem. 86, 685-714 (2017)

4. Pacheu-Grau, D. et al. Hum. Mol. Genet. 27, 4135-4144 (2018).

5. Webb, C. T., Gorman, M. A., Lazarou, M., Ryan, M. T. \& Gulbis, J. M. Mol. Cell 21, 123-133 (2006)

6. Baker, M. J. et al. Mol. Biol. Cell 20, 769-779 (2009).

7. Weinhaupl K. et al. bioRxiv. https://doi.org/10.1101/2020.03.13.990150 (2020).

8. Weinhaupl, K. et al. Cell 175, 1365-1379 (2018).

9. Kang, Y. et al. Elife 5, e46354 (2016).

10. Callegari, S. et al. FEBS Lett. 590, 4147-4158 (2016).

11. Kang, Y. et al. Mol. Cell 67, 457-470 (2017).

12. Vukotic, M. et al. Mol. Cell 67, 471-483 (2017).

13. Kumazaki, K. et al. Nature 509, 516-520 (2014).

14. Bektas, M. et al. J. Cell Biol. 169, 801-811 (2005).

15. Rehling, P. et al. Science 299, 1747-1751 (2003). 